\title{
FISIOPATOLOGIA DA ENXAQUECA (OU MIGRÂNEA)
}

\author{
THE PATHOPHYSIOLOGY OF MIGRAINE
}

Maurice Vincent

Professor Adjunto da Faculdade de Medicina da Universidade Federal do Rio de Janeiro. Serviço de Neurologia, Hospital Universitário Clementino Fraga Filho.

CoRRESPondÊnCIA: Av. das Américas, 1155/504 - CEP: 22631-000 - Rio de Janeiro - Fone: (021)4399245 Fax: (021)4943648 E-mail: vincent@unisys.com.br

VINCENT M. Fisiopatologia da enxaqueca (ou migrânea). Medicina, Ribeirão Preto, 30: 428-436, out./dez. 1997.

RESUMO: A fisiopatologia da enxaqueca tem sofrido uma evolução considerável, principalmente nos últimos quinze anos. O conhecimento dos mecanismos genéticos, incluindo a participação do gene CACNL1A4 no cromossoma 19p13.1, que codifica a subunidade $\alpha 1$ de um canal de cálcio, tipo $P / Q$, específico do cérebro, dos mediadores e neurotransmissores envolvidos com a inflamação neurogênica, tais como o Peptídeo Relacionado ao Gene da Calcitonina (CGRP), a NK-1 e a SP; a influência do NO como molécula algógena fundamental na enxaqueca; o desenvolvimento de modelos experimentais, incluindo o modelo do extravasamento de plasma, conforme desenvolvido pelo grupo do Moskowitz; e novas técnicas de neuroimagem (PET, MR e MEG) são alguns exemplos dos avanços alcançados. Isto tem propiciado o desenvolvimento de novos medicamentos antienxaquecosos. A depressão alastrante de Leão tem sido confirmada como o fenômeno neurofisiológico mais importante na enxaqueca: ativa o sistema trigeminovascular, induz vasodilatação mediada por CGRP e a expressão de c-fos no núcleo do trigêmio, no tronco cerebral. A dor na migrânea não é secundária à vasodilatação, o que não mais justifica a divisão das cefaléias em "vasculares" e "não vasculares".

UNITERMOS: Enxaqueca; fisiopatologia.

\section{INTRODUÇÃO}

Muitas têm sido as hipóteses propostas para explicar a origem das enxaquecas, tais como alimentos, alergias, vasoespasmos, alterações serotoninérgicas, desordens plaquetárias, problemas na barreira hematoencefálica, origem psicogênica, entre outras. As antigas teorias "vascular" e "neurogênica" podem hoje ser consideradas em conjunto, pois não são mutualmente exclusivas.

Antigamente, as cefaléias eram divididas em "vasculares" e "não vasculares"(1). Entretanto, nem todos os pacientes com afecções cerebrovasculares experimentam dor ${ }^{(2,3)}$, e nem sempre a vasodilatação é dolorosa. A hipótese de a aura surgir por vasocons- tricção e de a dor enxaquecosa originar-se na vasodilatação, desde os estudos de $\operatorname{Wolff}^{(4,5)}$, parece simplista demais. Tal teoria, adequada à luz do conhecimento e da tecnologia da época, passou a ser questionada no início da década passada ${ }^{(6)}$. Olesen et al. usaram ${ }^{133} \mathrm{Xe}$ intracarotídeo e duzentos e cinquenta e quatro detectores externos, acoplados ao crânio, para medir, de maneira mais precisa, o fluxo sangüíneo cerebral regional (rCBF) em pacientes com ataques de enxaqueca, encontrando uma redução durante a aura. Tal redução parecia iniciar de maneira restrita, no pólo occipital, avançando progressivamente para outras regiões. Nem sempre, porém, a hipoperfusão se iniciava nos pólos occipitais. Alguns pacientes com aura não relacionada a fenômenos visuais tiveram hipoperfusão primeira- 
mente no lobo frontal ${ }^{(7)}$. Esse fenômeno, que, aparentemente, não respeitava os limites dos territórios vasculares, foi denominado spreading oligoemia ${ }^{(8)}$ e, posteriormente, spreading hypoperfusion $(\mathrm{SH})^{(10)}$.

$\mathrm{O}$ fato de a SH espalhar-se pelo encéfalo, sem respeitar a anatomia dos territórios vasculares, favorece a existência de um mecanismo neuronal subjacente, e não um fenômeno primariamente vascular. Curiosamente, a velocidade de propagação da SH era surpreendentemente semelhante à velocidade de um fenômeno conhecido como "Depressão Alastrante (DA) de Leão" ${ }^{(11,12,13)}$. Admite-se que a SH represente a repercussão hemodinâmica da passagem da DA.

Recentemente, Woods et al. tiveram a oportunidade de registrar e comprovar o fenômeno de $\mathrm{SH}$ em uma mulher que, por acaso, desenvolveu uma crise de enxaqueca espontânea, enquanto era submetida a estudos de perfusão cerebral, regional, por $\operatorname{PET}^{(14)}$. Este trabalho fornece uma clara evidência, ainda que indireta, da ocorrência de DA no cérebro humano, durante a crise de enxaqueca.

O Sumatriptan, um agonista serotoninérgico $5-\mathrm{HT}_{1 \mathrm{~B} / \mathrm{D}}$, utilizado contra crises de enxaqueca e cefaléia em salvas, tem propriedades vasoconstrictoras, e reduz a dor ao mesmo tempo em que o calibre dos vasos retorna ao normal ${ }^{(15)}$. Entretanto, seu mecanismo de ação pode não ser inteiramente vascular. Nós evidenciamos, por exemplo, que o Sumatriptan produz vasodilatação em artérias oftálmicas suínas ${ }^{(16)}$. Na cefaléia em salvas ("Cluster headache"), uma síndrome caracterizada por ataques intensos de cefaléia unilateral de duração curta, associada a fenômenos autonômicos ipsilaterais, há grande vasodilatação ocular. Assim, é improvável que este medicamento exerça seu efeito exclusivamente por vasoconstricção.

Os vários fatores possivelmente envolvidos na fisiopatologia da enxaqueca são discutidos a seguir e estão resumidos na Tabela I.

Tabela I - Mecanismos fisiopatológicos na enxaqueca

- Depressão alastrante

- Ativação do sistema trigeminovascular

- Inflamação neurogênica

- Vasodilatação indicada por - óxido nítrico

- serotonina

- Distúrbios do metabolismo energético

- Predisposição genética

\section{DEPRESSÃO ALASTRANTE}

Lashly, estudando sua própria aura visual, concluiu que as alterações seriam produzidas por alguma coisa que se propagava pelo córtex visual primário, a uma velocidade de aproximadamente $3 \mathrm{~mm} / \mathrm{min}^{(17)}$. Pouco depois, em 1944, estudando eletrofisiologia no córtex de coelhos, em Harvard, EUA, o Professor Aristides Leão, do Instituto de Biofísica da Universidade Federal do Rio de Janeiro, observou um fenômeno de depressão da atividade elétrica que, quando experimentalmente induzido, propagava-se pelo córtex em todas as direções ${ }^{(11)}$. Esse fenômeno foi denominado "depressão alastrante (DA)", popularizado na literatura internacional como "spreading depression of Leão". Como a velocidade de propagação da DA era, curiosamente, semelhante à velocidade de propagação do fenômeno cortical que Lashly sugeria ser o responsável pela aura visual, Leão sugeriria, posteriormente, que a DA poderia estar relacionada à fisiopatologia da enxaqueca ${ }^{(12)}$.

Moskowitz et al. demonstraram que a passagem da depressão alastrante provocava a expressão de $c$-fos - um marcador não específico da ativação neuronal - no núcleo do trigêmio ${ }^{(18)}$. A passagem da DA, portanto, ativa o sistema trigeminovascular. A dilatação dos vasos arteriais, na pia "mater" de gatos, ocorre após a indução de DA ${ }^{(19)}$, um efeito produzido, ainda que parcialmente, pelo CGRP. Entretanto, a passagem da DA não levou à detecção de CGRP em amostras extraídas da jugular de gatos ${ }^{(20)}$.

A DA é a possibilidade mais atraente para explicar a aura enxaquecosa ${ }^{(21,22)}$, com o que concordamos. Através de métodos recentes de neuroimagem, têm sido acumuladas evidências que reforçam o envolvimento da DA na fisiopatologia da enxaqueca, tanto com aura, quanto sem aura ${ }^{(23)}$. A participação da DA na fisiopatologia da enxaqueca, entretanto, não é universalmente aceita $^{(24)}$. Um dos argumentos mais fortes contra o envolvimento da DA na enxaqueca tem sido a falta de uma demonstração inquestionável de sua existência em humanos, in vivo. Recentemente, porém, evidências, sugerindo a presença do fenômeno, foram obtidas no córtex do lobo frontal direito de um paciente em coma, após traumatismo crânioencefálico ${ }^{(25)}$.

\section{SISTEMA TRIGEMINOVASCULAR}

Três tipos de fibras nervosas existem na parede dos vasos cranianos, sobretudo entre a adventícia e a média e na adventícia ${ }^{(26 / 30)}$. Essas fibras apresentam varicosidades, no interior das quais existem vesículas, 
contendo substâncias neurotransmissoras vasoativas, que são liberadas, quando da passagem do estímulo nervoso. Esses neurotransmissores interagem com substâncias vasorreguladoras presentes no sangue e/ou no vaso, liberadas pelo endotélio, e contribuem para a regulação do tono vascular.

As fibras simpáticas, originadas no gânglio cervical superior, contêm, além da noradrenalina (NA), o neuropeptídeo Y (NPY), um neurotransmissor com atividade vasoconstrictora ${ }^{(31)}$.

As fibras parassimpáticas, originadas no gânglio esfenopalatino, por sua vez, contêm acetilcolina (Ach), seu transmissor, vasodilatador, endoteliodependente, além de outros elementos, tais como o peptídeo intestinal vasoativo (VIP), também vasodilatador ${ }^{(32)}$.

As fibras sensitivas trigeminais amielínicas do tipo C, a partir do gânglio de Gasser, funcionam também como eferentes. Seus neurotransmissores e neuromoduladores incluem a Substância P (SP) o peptídeo relacionado ao gene da calcitonina (CGRP), além de outras taquicininas, como a Neurocinina A (NKA). A SP é um peptídeo que provoca vasodilatação mais modesta e relativamente mais curta, podendo atuar como neuromodulador do tono vascular ${ }^{(33)}$. Aumenta a permeabilidade vascular, mas não quebra a barreira hematoencefálica ${ }^{(34)}$. Não excita terminais nociceptores diretamente, embora condicione sua resposta a outros mediadores. O CGRP é um dos mais potentes vasodilatadores que se conhece. Existe em abundância na divisão oftálmica do trigêmio e causa vasodilatação relativamente intensa e longa ${ }^{(35,36)}$. O CGRP também pode ter efeito neuromodulador. Embora não provoque extravasamento protéico por si só, pode aumentá-lo, facilitando a ação da $\mathrm{SP}^{(37)}$.

O papel dessas fibras, no desenvolvimento da crise de enxaqueca, parece de particular importância( ${ }^{(38)}$. O chamado sistema trigêminovascular ${ }^{(39,40,41)}$ constitui o substrato anatomofuncional sobre o qual a crise enxaquecosa se desenvolve, incluindo vasos (sobretudo artérias), e estruturas trigeminais centrais e periféricas.

\section{INFLAMAÇÃO NEUROGÊNICA}

Moskowitz et al. ${ }^{(39)}$ desenvolveram um modelo experimental, em ratos, no qual o estímulo trigeminal induz, antidromicamente, vasodilatação e extravasamento de plasma, com liberação dos transmissores mencionados acima ${ }^{(39 / 43)}$. Este fenômeno é chamado inflamação neurogênica.

A primeira evidência da liberação de CGRP, durante ataques de enxaqueca, foi obtida no sangue jugular ipsilateral à dor, em pacientes enxaqueco- $\operatorname{sos}^{(38,44)}$. Em animais, a estimulação trigeminal leva à liberação de neuropeptídeos como a SP e o CGRP ${ }^{(45,46)}$. O CGRP e a SP podem ser considerados "marcadores" da atividade trigeminal. Sua detecção indica ativação do sistema trigemiovascular, tal ocorre também na cefaléia em salvas ${ }^{(47,48)}$.

A inflamação neurogênica constitui um modelo experimental para enxaquecas ${ }^{(49)}$. Resultados experimentais preliminares, porém, podem não ser paralelos a respostas clínicas correspondentes. O RPR100893, um antagonista de SP, na dose de $20 \mathrm{mg}$ VO, mostrou-se ineficaz contra a enxaqueca, embora experimentalmente bloqueasse, de forma eficiente, a inflamação neurogênica $^{(50)}$. O mesmo ocorreu com o Bosentan (RO470232, $250 \mathrm{mg}$ EV), um antagonista da Endotelina ${ }^{(51)}$.

\section{5. ÓXIDO NÍTRICO}

Algumas substâncias vasodilatadoras não atuam no músculo liso, vascular, diretamente, mas induzem à liberação, pelo endotélio, do outrora chamado EDRF ("Endothelial derived relaxing factor") ${ }^{(52)}$, Evidenciou-se, posteriormente, que o EDRF é o óxido nítrico $(\mathrm{NO})^{(53,54)}$, sintetizado a partir da L-arginina pela NOS ("Nitric Oxide Synthase")(55).

O NO é também um neurotransmissor, presente em fibras nervosas, inclusive as perivasculares ${ }^{(56,57)}$. Olesen et al. cogitaram a possibilidade do NO constituir uma importante molécula algógena na enxaque$\mathrm{ca}^{(58,59)}$, pois:

1) a ativação da via NO-cGPM causa crises de enxaqueca nos enxaquecosos, ataques de cefaléia em salvas, em pacientes com cefaléia em salvas, e cefaléias não específicas, em outras pessoas;

2) drogas que são eficientes no tratamento da enxaqueca e de outras cefaléias vasculares, que não sejam analgésicos gerais, exercem sua atividade, inibindo uma ou mais etapas da via NO-cGMP, ou antagonizando os efeitos dos metabólitos gerados por esta via;

3) substâncias que podem causar um ataque de enxaqueca, como a nitroglicerina, fazem-no, ativando a via NO-cGMP, ou agindo como agonistas das substâncias em uma ou mais etapas desta via.

É provável que o NO neuronal tenha menor importância na crise enxaquecosa, pois a administração de um inibidor não seletivo da NOS, como o L-NAME - que atua tanto na NOS do nervo quanto na do endotélio -, bloqueia o extravasamento de plasma no modelo de inflamação neurogênica, ao passo que um inibidor seletivo da NOS neuronal - 7-nitroindazole - é, comparativamente, ineficaz ${ }^{(60)}$. Curiosa- 
mente, a DA produz uma "up-regulation" da NOS endotelial, em ratos ${ }^{(61)}$, podendo justificar, em parte, uma alteração da susceptibilidade à dor, em pacientes enxaquecosos.

\section{SEROTONINA (5-HIDROXITRIPTAMINA - 5-HT)}

Sicuteri demonstrou haver maior quantidade de ácido 5-hidroxiindolacético (5-HIAA), um metabólito da 5-HT, na urina de pacientes enxaquecosos ${ }^{(62)}$. Desde então, muitas especulações foram feitas a respeito do papel da 5-HT nas cefaléias ${ }^{(63 / 67)}$.

Os receptores para a 5-HT, numerados de 1 a $7^{(68,69,70)}$, são constituídos por cinco subtipos: A, B, D, E e F. O receptor 5-HT ${ }_{1 \mathrm{D}}$ é dividido por dois outros subtipos, o receptor 5-HT ${ }_{1 \mathrm{D} \alpha}$, localizado pré-juncionalmente nas fibras sensitivas, nervosas, perivasculares, e o receptor $5-\mathrm{HT}_{1 \mathrm{D} \beta}$, pós-juncional, presente no músculo liso, vascular ${ }^{(71)}$. O receptor $5-\mathrm{HT}_{1 \mathrm{D} \alpha}$, passou a ser chamado 5-HT $1 \mathrm{D}$ e o receptor $5-\mathrm{HT}_{1 \mathrm{D} \beta}$ passou a ser simplesmente $5-\mathrm{HT}_{1 \mathrm{~B}}$.

O Sumatriptan, uma eficiente droga antienxaquecosa específica, tem atividade agonista, em alguns receptores da família 1, com maior afinidade pelos sub-receptores $1 \mathrm{D}$ e $1 \mathrm{~B}\left(5-\mathrm{HT}_{1 \mathrm{~B} / \mathrm{D}}\right)$, cuja ativação leva à vasoconstricção ${ }^{(72)}$, e pelo receptor $\mathrm{F}$.

A ativação do receptor $5-\mathrm{HT}_{1 \mathrm{~B}}$ provoca vasoconstricção. Essa tem sido uma das explicações para o mecanismo de ação do Sumatritran e outros medicamentos relacionados ${ }^{(72,73,74)}$. Como existem receptores $5-\mathrm{HT}_{1 \mathrm{D}}$, situados pré-juncionalmente, para os quais o Sumatriptan também tem atividade agonista, existe a possibilidade de que a droga atue na crise enxaquecosa, não por vasoconstricção ${ }^{(75 / 78)}$, mas bloqueando a inflamação neurogênica.

A CP122.288 é uma substância com potência mil vezes maior que a do Sumatriptan, no bloqueio da inflamação neurogênica e extravasamento de plasma, sem induzir vasoconstricção ${ }^{(79,80)}$. Medicamentos com essas propriedades poderão representar armas importantes contra as enxaquecas, no futuro.

Sítios de ligação para o Sumatriptan, no sistema nervoso central, foram mapeados em animais ${ }^{(81)} \mathrm{e}$ no homem ${ }^{(82,83)}$. As maiores concentrações de receptores para o Sumatriptan estão, em ordem decrescente, nos córtex visual e frontal (camadas IV e V), no núcleo do trato solitário e espinhal do trigêmio, além de em outros locais. Embora o Sumtriptan não ultrapasse facilmente a barreira hematoencefálica ${ }^{(34)}$, é possível que, durante a crise enxaquecosa, a droga alcance algum receptor central, cuja ativação tenha importância no seu mecanismo de ação. Curiosamente, há muitos receptores no córtex visual, região freqüentemente envolvida na enxaqueca com aura, particularmente rica em receptores $5-\mathrm{HT}_{1 \mathrm{~F}}$. Embora o Sumatriptan seja considerado um medicamento ineficaz contra a aura ${ }^{(84)}$, é possível que tenha algum efeito nessa fase ${ }^{(85)}$. Além disto, o Sumatriptan bloqueia a DA, reversivelmente e de maneira dose-dependente, em retinas isoladas de pintos ${ }^{(86)}$, embora não tenha sido demonstrada por outros autores qualquer interferência de substâncias antienxaquecosas na DA, em gatos $^{(87)}$. Sítios de ligação no núcleo do trato solitário e no núcleo do trigêmio podem estar relacionados aos efeitos antiemético e antiálgico do Sumatriptan.

O Sumatriptan tem, portanto, três possibilidades de atuação: na musculatura lisa da parede vascular - pós-juncionalmente; na fibra sensitiva perivascular - pré-juncionalmente - e no sistema nervoso central. Quanto à última possibilidade, foi demonstrado que o Zolmitriptan (311C90), uma substância análoga, que cruza melhor a barreira hematoencefálica, quando administrado sistemicamente, inibe experimentalmente a atividade trigeminovascular, por ação central ${ }^{(88)}$. Além disto, o Zolmitriptan interfere com potenciais evocados, auditivos, relacionados de maneira inversa à produzida pela transmissão serotoninérgica ${ }^{(89)}$.

\section{MÉTODOS DE NEUROIMAGEM}

Os exames rotineiros de imagem produzem resultados normais nas enxaquecas. Tomografias computadorizadas, ressonâncias magnéticas nucleares, e, até mesmo, arteriografias cerebrais têm sido, desnecessariamente, realizadas em pacientes com enxaqueca.

Métodos mais sofisticados, porém, podem auxiliar na investigação da fisiopatologia da enxaqueca. Muitos trabalhos têm demonstrado alterações do fluxo sanguiíneo cerebral regional. Isso inclui o trabalho de Woods et al. ${ }^{(14)}$; de Weiller et al ${ }^{(90)}$, no qual se demonstrou, pela primeira vez, a provável existência de um centro para enxaqueca, no tronco cerebral; e o de Sorensen et al. (APUD Flippen \& Welch $^{(91)}$, estudando ataques espontâneos de enxaqueca, através de técnicas de ressonância magnética.

A magnetoencefalografia (MEG) evidencia alterações exclusivas dos enxaquecosos ${ }^{(92)}$. As ondas "slow field changes" foram semelhantes às observadas durante experimentos, induzindo-se DA em coelhos, e as "large amplitude waves" (LAW) representariam despolarizações neuronais, focais, cujo alastramento é limitado pela descontinuidade dos sulcos corticais. Tais dados constituem outra evidência indireta da presença da DA em humanos. 
A espectroscopia por ressonância magnética é um método de imagem que permite se averiguem parâmetros metabólicos e energéticos, in vivo. A espectrometria por fósforo $\left({ }^{31} \mathrm{P}-\mathrm{MRS}\right)$ tem sido estudada em pacientes com enxaqueca ${ }^{(93 / 96)}$, demonstrando a provável existência de uma desordem do metabolismo energético, talvez de origem mitocondrial. Os dados sugerem perda de fosfatos de alta energia (PCr) e aumento dos fosfatos de pequena energia ( $\mathrm{Pi})$, sem alteração nos níveis de ATP. Tais alterações, detectadas principalmente nas regiões mais rostrais do cérebro (frontal e frontotemporal), foram mais marcadas nos pacientes com enxaqueca associada a aura.

\section{ENXAQUECA E GENÉTICA}

Sabe-se que a enxaqueca, sobretudo a associada a aura ${ }^{(97)}$, tem influência genética, talvez autossômica recessiva, com penetrância reduzida ${ }^{(98,99)}$. Estudos em gêmeos têm confirmado a importância da influência genética na enxaqueca ${ }^{(100,101)}$.

Uma forma curiosa de doença cerebrovascular, familiar, denominada "CADASIL" (Cerebral Autosomal Dominat Arteriopathy with Subcortical Infarcts and Leucoencephalopathy) foi identificada recentemente, estando sua ocorrência ligada ao cromossoma 19p12 ${ }^{(102)}$. Pacientes com CADASIL são mais freqüentemente acometidos por enxaqueca com aura do que a população geral ${ }^{(102,103,104)}$. Aquele cromossoma também está envolvido na transmissão da "enxaqueca hemiplégica, familiar" (FHM) ${ }^{(105,106)}$. Quatro mutações diferentes na subunidade $\alpha 1$ de um canal de cálcio, voltagem-dependente, tipo $\mathrm{P} / \mathrm{Q}$, específico do cérebro, mapeadas no gene CACNL1A4, no cromossoma 19p13.1, foram identificadas em quatro famílias com $\mathrm{FHM}^{(107)}$. Diante das evidências genéticas, é possível que a enxaqueca seja classificada, no futuro, como uma "channelopathy" (ou desordem canalar).

\section{UMA EXPLICAÇÃO INTEGRADA PARA A OCORRÊNCIA DA CRISE DE ENXAQUECA}

A alteração genética de um canal de cálcio cerebral, específico provoca um estado de hiperexcitabilidade que torna o SNC mais susceptível a estímulos externos (luminosos, alimentares, dentre outros) e internos (estresse emocional, por exemplo) e ao aparecimento de DA. Áreas específicas, na porção média do tronco cerebral, tornam-se particularmente excitáveis, podendo funcionar como centros da enxaqueca. Náuseas e vômitos podem se ori- ginar da excitação do núcleo do trato solitário. O conseqüente surgimento do fenômeno de depressão alastrante, no córtex cerebral, ativa o sistema trigeminovascular, tanto nos vasos da periferia, quanto nos do núcleo trigeminal, no tronco cerebral. A DA pode ser subclínica, provocando, nesse caso, a enxaqueca sem aura. O tipo de aura depende da(s) área(s) cortical(ais) atingida(s). Com a conseqüente ativação do sistema trigeminovascular, há liberação de substâncias neurotransmissoras, vasodilatadoras, como o CGRP e a SP, que interagem com outras substâncias liberadas localmente pelo próprio vaso e por fibras nervosas simpáticas e parassimpáticas. A excitação do sistema trigeminal caminha antidromicamente, pelas redes perivasculares, difundindo o processo de inflamação neurogênica. Ortodromicamente, os estímulos trigeminais atingem o tálamo e, posteriormente, o córtex cerebral, originando a sensação de dor.

Esta hipótese integrativa de fisiopatologia da enxaqueca está mostrada na Figura 1.

A enxaqueca não é dor, mas um estado de susceptibilidade a crises que se caracterizam por um complexo de sintomas que podem incluir cefaléia. O que se considera como "causas" de enxaqueca (estresse, chocolate, vinho, alimentos com tiramina, alterações temporomandibulares, entre outras) são, na verdade, simples fatores desencadeantes, que dependem de uma predisposição individual para o desencadeamento da crise.

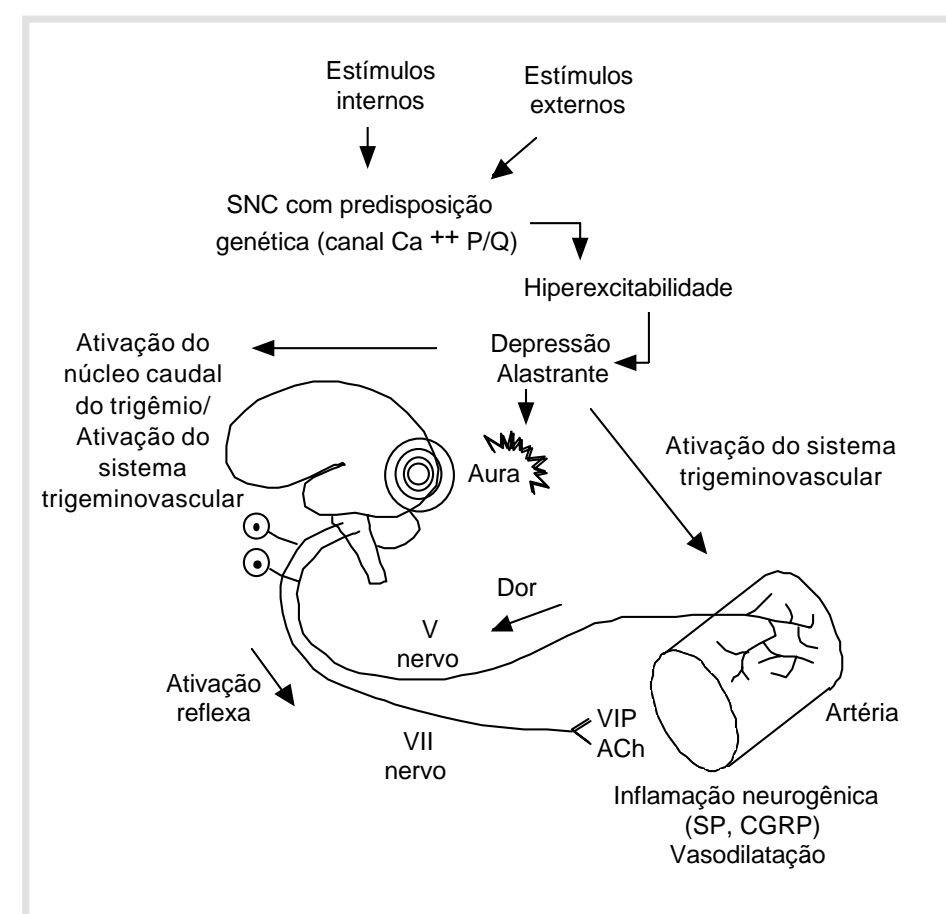

Figura 1 - Mecanismos fisiopatológicos na enxaqueca. 
VINCENT M. The pathophysiology of migraine. Medicina, Ribeirão Preto, 30: 428-436, oct./dec. 1997.

ABSTRACT: The knowledge of the pathophysiology of migraine has advanced to a great extent, particularly over the last 15 years. New genetic data, involving mutations in the CACNL1A4 gene on chromosome 19p13.1 encoding the a1 subunit of the brain specific P/Q-type calcium channel, the role of neuropeptides such as CGRP, NK1 and SP, the influence of NO as a key molecule in migraine, the development of experimental models including the plasma extravasation model developed by Moskowitz' group and the use of advanced neuroimaging techniques (PET, MR and MEG) are some of the recent achievements. New anti-migraine drugs are on the way, developed according to the new pathophysiological knowledge. Leão's spreading depression remains as one of the most important neurophysiological phenomena in migraine. It activates the trigeminovascular system, induces CGRP-mediated vasodilation and provokes the expression of $c$-fos in the trigeminal nucleus caudalis. The migrainous pain does not seem to be produced by vasodilation, so classifying headaches in "vascular" and "non-vascular" is no longer appropriate.

UNITERMS: Migraine; physiopathology.

\section{REFERÊNCIAS BIBLIOGRÁFICAS}

1 - AD HOC COMMITTEE ON CLASSIFICATION OF HEADACHE. Classification of headache. JAMA 179: 717-718, 1962.

2 - ANDRÉ C; NEVES FF \& VINCENT MB. Headache in TIA. Funct Neurol 11: 195-200, 1996

3 - ANDRÉ C \& VINCENT MB. On the pathophysiology of headache following TIA. Arq Neuropsiquiatr 55: 167-173, 1997.

4 - GRAHAM JG \& WOLFF HG. Mechanism of migraine headache and action of ergotamine tartrate. Arch Neurol Psychiatry 39: 737-763, 1938.

5 - RAY BS \& WOLFF HG. Experimental studies on headache. Pain sensitive structures of the head and their significance in headache. Arch Surg 41: 813-856, 1940.

6 - OLESEN $\mathrm{J}$ et al. The common migraine attack may not be initiated by cerebral ischaemia. Lancet 2: 438-440, 1981.

7 - FRIBERG $L$ et al. Focal ischemia caused by instilation of cerebrovascular tone during attacks of hemiplegic migraine. Brain 110: 917-934, 1987.

8 - LAURITZEN M et al. The changes of regional cerebral blood flow during the course of classical migraine attacks. Ann Neurol 13: 633-641, 1983.

9 - LAURITZEN M \& OLESEN J. Regional cerebral blood flow during migraine attacks by xenon-133 inhalation and emission tomography. Brain 107: 447-461, 1984.

10 - OLESEN J, LARSEN B \& LAURITZEN M. Focal hyperemia followed by spreading oligemia and impaired activation of rCBF in classic migraine. Ann Neurol 9: 344-352, 1981.

11 - LEÃO AAP. Spreading depression of activity in cerebral córtex. J Neurophysiol 7: 359-390, 1944.

12 - LEÃO AAP \& MORISON RS. Propagation of spreading cortical depression. J Neurophysiol 8: 33-45, 1945.
13 - LEÃO AAP. On the inferred relationship of migraine and spreading depression. In: CLIFFORD ROSE F, ed Advances in headache research. John Libbey, Chicago, p. 19-24, 1987.

14 - WOODS RP; IACOBONI M \& MAZZIOTTA JC. Bilateral spreading cerebral hypoperfusion during spontaneous $\mathrm{mi}-$ graine headache. N Engl J Med 331: 1689-1692, 1994.

15 - FRIBERG LET al. Migraine pain associated with middle cerebral artery dilatation: reversal by sumatriptan. Lancet 338:13-17, 1991.

16 - VINCENT MB et al. Sumatriptan relaxes isolated porcine ophthalmic artery, but inhibits VIP-induced relaxation. Cephalalgia 13: 378-382, 1993.

17 - LASHLEY KS. Patterns of cerebral integration indicated by the scotomas of migraine. Arch Neurol Psychiatry 46: 259-264, 1941

18 - MOSKOWITZMA; NOZAKI K \& KRAIG RP. Neocortical spreading depression provokes the expression of c-fos protein-like immunoreactivity within trigeminal nucleus caudalis via trigeminovascular mechanisms. J Neurosci 13: 1167-1177, 1993.

19 - WAHL M et al. Involvement of calcitonin gene-related peptide (CGRP) and nitric oxide (NO) in the pial artery dilatation elicited by cortical spreading depression. Brain Res 637: 204-210, 1994.

20 - PIPER RD et al. Cortical spreading depression does not result in the release of calcitonin gene-related peptide into the external jugular vein of the cat: relevance to human migraine. Cephalalgia 13:180-183, 1993.

21 - LAURITZEN M. Spreading depression and migraine. Pathol Biol 40: 332-337, 1992.

22 - LAURITZEN M. Cortical spreading depression as putative migraine mechanism. Trends Neurosci 10: 447-467, 1987.

23 - BOUCKOMS AJ et al. Trigeminal facial pain: a model of peptides and monoamines in intracerebral cerebrospinal fluid. Agressologie 32: 271-274, 1991. 
24 - INGVARDSEN BK et al. Cortical spreading depression does not activate trigeminal C fibres. In: OLESEN J \& EDVINSSON $L$, eds.Headache pathogenesis monoamines, neuropeptides, purines, and nitric oxide. Lippincott; Raven Press, Philadelphia: p. 117-122, 1997 (Frontiens in headache research, v. 7).

25 - MAYEVSKY A et al. Cortical spreading depression recorded from the human brain using a multiparametric monitoring system. Brain Res 740: 268-274, 1996.

26 - EDVINSSON L. Innervation and effects of dilatory neuropeptides on cerebral vessels. New aspects. Blood Vessels 28: 35-45, 1991.

27 - EDVINSSON L. Innervation and effects of dilatory neuropeptides on cerebral vessels. Blood Vessels 28: 35-45, 1991.

28 - MIONE MC; RALEVIC V \& BURNSTOCK G. Peptides and vasomotor mechanisms. Pharmacol Ther 46: 429-468, 1990.

29 - WALTERS BB; GILLESPIE SA \& MOSKOWITZ MA. Cerebrovascular projections from the sphenopalatine and otic ganglia to the middle cerebral artery of the cat. Stroke 17: 488-494, 1986.

30 - UDDMAN R \& EDVINSSON L. Neuropeptides in the cerebral circulation. Cerebrovasc Brain Metab Rev 1: 230-252, 1989.

31 - EDVINSSON L et al. Nerve fibres containing neuropeptide $Y$ in the cerebrovascular bed: immunocytochemistry, radioimmunoassay, and vasomotor effects. J Cereb Blood Flow Metab 7: 45-57, 1987

32 - DUCLES SP \& SAID SI. Vasoactive intestinal polypeptide as a neurotransmitter in the cerebral circulation. Eur $\mathbf{J}$ Pharmacol 78: 371-374, 1982.

33 - VINCENT MB et al. Substance $P$ augments the rate of vasodilation induced by calcitonin gene-related peptide in porcine ophthalmic artery in vitro. Neuropeptides 22: 137-141, 1992.

34 - HUMPHREY PP et al. Preclinical studies on the anti-migraine drug, sumatriptan. Eur Neurol 31: 282-290, 1991.

35 - BAKKEN IJ et al. Mutual modification of vasoactivity by calcitonin gene-related peptide and endothelin-1 in isolated porcine ophthalmic artery. Neuropeptides 23: 209-214, 1992.

36 - BAKKEN IJ et al. Vasodilation in porcine ophthalmic artery: Peptide interaction with acetylcholine and endothelial dependence. Neuropeptides 29: 69-75, 1995.

37 - LE GREVES $P$ et al. Calcitonin gene related peptide is a potent inhibitor of substance P degradation. Eur J Pharmacol 115: 309-311, 1985.

38 - EDVINSSON L \& GOADSBY P. Neuropeptides in migraine and cluster headache. Cephalalgia 14: 320-327, 1994.

39 - MOSKOWITZ MA et al. Neurotransmitters and the fifth cranial nerve: is there a relation to the headache phase of $\mathrm{mi}-$ graine? Lancet 2: 883-884, 1979.

40 - MOSKOWITZ MA. The neurobiology of vascular head pain. Ann Neurol 16:157-168, 1984.
41 - BUZZI MG \& MOSKOWITZ MA. The trigemino-vascular system and migraine. Pathol Biol 40: 313-317, 1992.

42 - MARKOWITZ S; SAITO K \& MOSKOWITZ MA. Neurogenically mediated leakage of plasma protein occurs from blood vessels in dura mater but not brain. J Neurosci 7: 4129-4136, 1987.

43 - MAYBERG MR; ZERVAS NT \& MOSKOWITZ MA. Trigeminal projections to supratentorial pial and dural blood vessels in cats demonstrated by horseradish peroxidase histochemistry. J Comp Neurol 223: 46-56, 1984.

44 - GOADSBY PJ; EDVINSSON L \& EKMAN R. Vasoactive peptide release in the circulation of humans during migraine headaches. Ann Neurol 28: 183-187, 1990.

45 - GOADSBY PJ; EDVINSSON L \& EKMAN R. Release of vasoactive peptides in the external circulation of humans and the cat during activation of the trigeminovascular system. Ann Neurol 23: 193-196, 1988.

46 - BUZZI MG et al. Dihydroergotamine and sumatriptan attenuate levels of CGRP in plasma in rat superior sagittal sinus during electrical stimulation of the trigeminal ganglion. Neuropharmacology 30: 1193-1200, 1991.

47 - GOADSBY PJ \& EDVINSSON L. Human in vivo evidence for trigeminovascular activation in cluster headache. Neuropeptide changes and effects of acute attacks therapies. Brain 117: 427-434, 1994.

48 - GOADSBY PJ \& EDVINSSON L. Evidence of trigeminovascular activation in man during acute cluster headache. Cephalalgia 13: 30, 1993. (Abstract) Suppl 13.

49 - CONNOR HE \& BEATTIE DT. Migraine. More advanced experimental models for drug development. In: OLESEN J \& MOSKOWITZ MA, eds. Experimental headache models. Lippincott; Raven Press, Philadelphia, p. 19-26, 1995.

50 - DIENER HC, FOR THE RPR 100893/201 MIGRAINE STUDY GROUP. Substance P antagonist RPR 100893/201 is not effective in human migraine attacks. In: Abstract Sixth International Headache Research Seminar, Copenhagen, Denmark; 1995.

51 - MAY A et al. Endothelin antagonist bosentan blocks neurogenic inflamation, but is not effective in aborting migraine attacks. Pain 67: 375-378, 1996.

52 - MAUSKOP A. Trigeminal neuralgia (tic douloureux). J Pain Symptom Manage 8: 148-154, 1993.

53 - PALMER RMJ; FERRIGE AG \& MONCADA S. Nitric oxide release accounts for the biological activity of endotheliumderived relaxing factor. Nature 327: 524-526, 1987.

54 - IGNARRO LJ et al. Endothelium-derived relaxing factor produced and released from artery and vein is nitric oxide. Proc Natl Acad Sci 84: 9265-9269, 1987.

55 - MONCADA S; PALMER RMJ \& HIGGS EA. Nitric oxide: physiology, pathophysiology and pharmacology. Pharmacol Rev 43: 109-141, 1991.

56 - BREDT DS; HWANG PM \& SNYDER SH. Localization of nitric oxide synthase indicating a neural role for nitric oxide. Nature 347: 768-770, 1990. 
57 - MORRIS R et al. Nitric oxide may act as a messenger between dorsal root ganglion neurones and their satellite cells. Neurosci Lett 137: 29-32, 1992.

58 - IVERSEN HK \& OLESEN J. Nitroglycerin-induced headache is not dependent on histamine release: support for a direct nociceptive action of nitric oxide. Cephalalgia 14: 437-442s, 1994.

59 - OLESEN J et al. The nitric oxide hypothesis of migraine and other vascular headaches. Cephalalgia 15: 94-100, 1995.

60 - LIMMROTH V; CUTRER FM \& MOSKOWITZ MA. Neurotransmitters and neuropeptides in headache. Curr Opin Neurol 9: 206-210, 1996.

61 - CHOPP M et al. Nitric oxide synthase is induced in cerebral endothelial cells by spreading depression. Cephalalgia 13: 1-116, 1993. (Abstract). Suppl 13.

62 - SICUTERI F; TESTI A \& ANSELMI B. Biochemical investigations in headache: increase in hydroxyindoleacetic acid excretion during migraine attacks. Int Arch Allergy 19: 55-58, 1961.

63 - LANCE JW. History of involvement of 5-HT in primary headaches. In: OLESEN J \& SAXENA PR, eds. 5-hydroxytryptamine mechanisms in primary headaches. Raven Press, New York, p. 19-28, 1992.

64 - HUMPHREY PP et al. Serotonin and migraine. Ann N Y Acad Sci 600: 587-598, 1990.

65 - SILBERSTEIN SD. Serotonin (5HT) and migraine. Headache 34: 408-417, 1994.

66 - FERRARI MD et al. Serotonin metabolism in migraine. Neurology 39: 1239-1242, 1989.

67 - FERRARI MD \& SAXENA PR. On serotonin and migraine: a clinical and pharmacological review. Cephalalgia13: 151-165, 1993.

68 - BONATE PL. Serotonin receptor subtypes: functional, physiological, and clinical correlates. Clin Neuropharmacol 14: 1-16, 1991.

69 - BAEZ M et al. Molecular biology of serotonin receptors. Obesity Res 4: 441-447, 1996.

70 - HOYER D et al. International union of pharmacology classification of receptors for 5-hydroxytryptamine (serotonin). Pharmacol Rev 46: 157-203, 1994.

71 - HARTIG PR et al. Alignment of receptor nomenclature with the human genome: classification of the $5-\mathrm{HT}_{1 \mathrm{~B}} 5-\mathrm{HT}_{1 \mathrm{D}}$ receptors subtypes. Trends Pharmacol Sci 17: 103-105, 1996.

72 - JANSEN I et al. Sumatriptan is a potent vasoconstrictor of human dural arteries via a $5-\mathrm{HT}_{1}$-like receptor. Cephalalgia 12: 202-205, 1992.

73 - HUMPHREY PP \& FENIUK W. Mode of action of the antimigraine drug sumatriptan. Trends Pharmacol Sci 12: 444-446, 1991.
74 - HUMPHREY PPA \& GOADSBY PJ. The mode of action of sumatriptan is vascular? A debate. Cephalalgia 14: 401-410, 1994.

75 - BUZZI MG \& MOSKOWITZ MA. The antimigraine drug, sumatriptan (GR43175), selectively blocks neurogenic plasma extravasation from blood vessels in dura mater. $\mathbf{B r} \mathbf{J}$ Pharmacol 99: 202-209, 1990.

76 - BUZZI MG \& MOSKOWITZ MA. Evidence for 5-HT $\mathrm{HB}_{1 \mathrm{D} / \mathrm{D}}$ receptors mediating the antimigraine effect of sumatriptan and dihydroergotamine. Cephalalgia 11: 165-168, 1991.

77 - MOSKOWITZ MA. Neurogenic versus vascular mechanisms of sumatriptan and ergot alkaloids in migraine. Trends Pharmacol Sci 13: 307-311, 1992.

78 - NOZAKI K; MOSKOWITZ MA \& BOCCALINI P. CP-93129, sumatriptan, and dihydroergotamine block c-FOS expression within the rat trigeminal nucleus caudalis caused by chemical stimulation of the meninges. Br J Pharmacol 106: 409-415, 1992.

79 - LEE WS \& MOSKOWITZ MA. Conformationally restricyed sumatriptan analogues, CP-122,288 e CP-122,638 exhibit enhanced potency against neurogenic inflammation in dura mater. Brain Res 626: 303-305, 1993.

80 - BEATTIE DT \& CONNOR HE. The pre- and postjunctional activity of CP-122,288, a conformationally restricted analogue of sumatriptan. Eur J Pharmacol 276: 271-276, 1995.

81 - WAEBER C \& MOSKOWITZ MA. $\left[{ }^{3} H\right]$ Sumatriptan labels both $5-\mathrm{HT}_{1 \mathrm{D}}$ and $5-\mathrm{HT}_{1 \mathrm{~F}}$ receptor binding sites in the guineapig brain: an autoradiographic study. Naunyn Schmiedeberg's Arch Pharmacol 352: 263-275, 1995.

82 - PASCUAL $\mathrm{J}$ et al. $\left[{ }^{3} \mathrm{H}\right]$ Sumatriptan binding sites in human brain: regional-dependent labelling of $5-\mathrm{HT}_{1 \mathrm{D}}$ and $5-\mathrm{HT} \mathrm{T}_{1 \mathrm{~F}}$ receptors. Eur J Pharmacol 295: 271-274, 1996.

83 - PASCUAL $\mathrm{J}$ et al. Autoradiographic distribution of $\left[{ }^{3} \mathrm{H}\right]$ Sumatriptan-binding sites in post-mortem human brain. Cephalalgia 16: 317-322, 1996.

84 - BATES $D$ et al. Subcutaneous sumatriptan during the migraine aura. Neurology 44: 1587-1592, 1994.

85 - CLIFFORD ROSE F. Sumatriptan arrests migraine aura. Headache 32: 365-366, 1992.

86 - MARANHÃO-FILHO PA et al. Sumatriptan blocks spreading depression in isolated chick retina. Cephalalgia 1997; In Press.

87 - KAUBE H \& GOADSBY PJ. Anti-migraine compounds fail to modulate the propagation of cortical spreading depression in the cat. Eur Neurol 34: 30-35, 1994.

88 - GOADSBY PJ \& HOSKIN KJ. Inhibition of trigeminal neurons by intravenous administration of the serotonin (5-HT)1B/D receptor agonist zolmitriptan (311C90): are brain sites therapeutic target in migraine? Pain 67: 355-359, 1996.

89 - SCHOENEN J; PROIETTI CECCHINI A \& AFRA J. Evidence for a central effect of a 5-HT1D agonist, 311C90, from a study of the intensity dependence of cortical auditory evoked potential. Eur J Neurol 3: 88, 1996. Suppl 5. 
90 - WEILLER $C$ et al. Brain stem activation in human migraine attacks. Nature Med 1: 658-660, 1995.

91 - FLIPPEN C \& WELCH KMA. Imaging the brain of migraine sufferers. Curr Opin Neurol 10: 226-230, 1997.

92 - BARKLEY GL et al. Magnetoencephalographic studies of migraine. Headache 30: 428-434, 1990.

93 - WELCH KMA et al. Brain pH in migraine: an in vivo phosphorus-31 magnetic resonance spectroscopy study. Cephalalgia 8: 273-277, 1988.

94 - WELCH KMA et al. Preliminary observations on brain energy metabolism in migraine studied by in vivo phosphorus 31 NMR spectroscopy. Neurology 39: 538-541, 1989.

95 - MONTAGNA P; CORTELLI B \& BARBIROLI B. Magnetic resonance spectroscopy studies in migraine. Cephalalgia 14: 184-193, 1994

96 - BARBIROLLI B et al. Abnormal brain and muscle energy metabolism shown by ${ }^{13} \mathrm{P}$ magnetic resonance spectroscopy in patients affected by migraine with aura. Neurology 42: 1209-1214, 1992.

97 - RUSSELL MB \& OLESEN J. Increased familial risk and evidence of genetic factor in migraine. BMJ 311: 541-544, 1995.

98 - DEVOTO $\mathrm{M}$ et al. Segregation analysis of migraine in 128 families. Cephalalgia 6: 101-105, 1986.

$99-\mathrm{MOCHI} \mathrm{M}$ et al. Testing models for genetic determination in migraine. Cephalalgia 13: 389-394, 1993.
100 - HONKASALO ML et al. Migraine and concomitant symptoms among 817 adult twin pairs. Headache 35: 70-78, 1995.

101 - LARSSON B; BILLE B \& PEDERSON N. Genetic influence in headache: a Swedish study. Headache 35: 513-519, 1995.

102 - JUNG HH et al. Cerebral autosomal dominant arteriopathy with subcortical infarcts and leukoencephalopathy: a clinicopathological and genetic study in a Swiss family. $\mathbf{J}$ Neurol Neurosurg Psychiatry 59:138-143, 1995.

103 - VERIN M et al. New phenotype of the cerebral autosomal dominant arteriopathy mapped to chromosome 19: migraine as the prominent clinical feature. J Neurol Neurosurg Psychiatry 59: 579-585, 1995.

104 - CHABRIAT H et.al. Clinical spectrum of CADASIL: a study of 7 families. Lancet 346: 934-939, 1995.

105 - HEADACHE CLASSIFICATION COMMITTEE OF THE INTERNATIONAL HEADACHE SOCIETY. Classification and diagnostic criteria for headache disorders, cranial neuralgias and facial pain. Cephalalgia 8: 1-96, 1988. Suppl 7.

106 - TERWINDT GM et al. The quest for migraine genes. Curr Opin Neurol 10: 221-225, 1997.

Recebido para publicação em 05/11/97.

Aprovado para publicação em 10/12/97. 